\title{
NUMBERS OF SOLUTIONS OF EQUATIONS IN FINITE FIELDS
}

\author{
ANDRÉ WEIL
}

The equations to be considered here are those of the type

$$
a_{0} x_{0}^{n_{0}}+a_{1} x_{1}^{n_{1}}+\cdots+a_{r} x_{r}^{n_{r}}=b .
$$

Such equations have an interesting history. In art. 358 of the Disquisitiones [1 a $]^{1}$ Gauss determines the Gaussian sums (the so-called cyclotomic "periods") of order 3, for a prime of the form $p=3 n+1$, and at the same time obtains the numbers of solutions for all congruences $a x^{3}-b y^{3} \equiv 1(\bmod p)$. He draws attention himself to the elegance of his method, as well as to its wide scope; it is only much later, however, viz. in his first memoir on biquadratic residues [1b], that he gave in print another application of the same method; there he treats the next higher case, finds the number of solutions of any congruence $a x^{4}-b y^{4} \equiv 1(\bmod p)$, for a prime of the form $p=4 n+1$, and derives from this the biquadratic character of $2 \bmod p$, this being the ostensible purpose of the whole highly ingenious and intricate investigation. As an incidental consequence ("coronidis loco," p. 89), he also gives in substance the number of solutions of any congruence $y^{2} \equiv a x^{4}-b(\bmod p)$; this result includes as a special case the theorem stated as a conjecture ("observatio per inductionem facta gravissima") in the last entry of his Tagebuch [1c] $;^{2}$ and it implies the truth of what has lately become known as the Riemann hypothesis, for the function-field defined by that equation over the prime field of $p$ elements.

Gauss' procedure is wholly elementary, and makes no use of the Gaussian sums, since it is rather his purpose to apply it to the determination of such sums. If one tries to apply it to more general cases, however, calculations soon become unwieldy, and one realizes the necessity of inverting it by taking Gaussian sums as a starting point. The means for doing so were supplied, as early as 1827 , by Jacobi, in a letter to Gauss [2a] (cf. [2b]). But Lebesgue, who in 1837 devoted two papers $[3 \mathrm{a}, \mathrm{b}]$ to the case $n_{0}=\cdots=n_{r}$ of equation (1), did not

Received by the editors October 2, 1948; published with the invited addresses for reasons of space and editorial convenience.

${ }^{1}$ Numbers in brackets refer to the bibliography at the end of the paper.

${ }^{2}$ It is surprising that this should have been overlooked by Dedekind and other authors who have discussed that conjecture (cf. M. Deuring, Abh. Math. Sem. Hamburgischen Univ. vol. 14 (1941) pp. 197-198). 
succeed in bringing out any striking result. The whole problem seems then to have been forgotten until Hardy and Littlewood found it necessary to obtain formulas for the number of solutions of the congruence $\sum_{i} x_{i}^{n} \equiv b(\bmod p)$ in their work on the singular series for Waring's problem [4]; they did so by means of Gaussian sums. More recently, Davenport and Hasse [5] have applied the same method to the case $r=2, b=0$ of equation (1) as well as to other similar equations; however, as they were chiefly concerned with other aspects of the problem, and in particular with its relation to the Riemann hypothesis in function-fields, ${ }^{3}$ the really elementary character of their treatment does not appear clearly.

As equations of type (1) have again recently been the subject of some discussion (cf. e.g. [6]), it may therefore serve a useful purpose to give here a brief but complete exposition of the topic. This will contain nothing new, except perhaps in the mode of presentation of the final results, which will lead to the statement of some conjectures concerning the numbers of solutions of equations over finite fields, and their relation to the topological properties of the varieties defined by the corresponding equations over the field of complex numbers.

We consider equation (1) over a finite field $k$ with $q$ elements; the $a_{i}$ are in $k$, and not 0 ; the $n_{i}$ are integers, which we assume to be $>0$ (only trifling modifications would be required if some were $<0)$. We shall first discuss the case $b=0$.

Let therefore $N$ be the number of solutions in $k$ of the equation

$$
a_{0} x_{0}^{n_{0}}+\cdots+a_{r} x_{r}^{n_{r}}=0 .
$$

For each $i$, let $d_{i}=\left(n_{i}, q-1\right)$ be the g.c.d. of $n_{i}$ and $q-1$; for each $i$ and for each $u$ in $k$, let $N_{i}(u)$ be the number of solutions of the equation $x^{n_{i}}=u ; N_{i}(u)$ is 1 for $u=0$, and is otherwise equal to $d_{i}$ or to 0 according as $u$ is or is not a $d_{i}$ th power in $k$. Put $L(u)=\sum_{i=0}^{r} a_{i} u_{i}$; we have

$$
N=\sum_{L(u)=0} N_{0}\left(u_{0}\right) \cdots N_{r}\left(u_{r}\right),
$$

where the sum is taken over all sets of values for the $u_{i}$ satisfying $L(u)=0$, or, as we may say, over all points $(u)=\left(u_{0}, \cdots, u_{r}\right)$ in the

${ }^{3}$ As to this, cf. H. Hasse, J. Reine Angew. Math. vol. 172 (1935) pp. 37-54. I regret that I did not quote either of these papers, where the connection between various kinds of exponential sums and the Riemann hypothesis is quite clearly expressed, in my recent note on the same subject, Proc. Nat. Acad. Sci. U.S.A. vol. 34 (1948) pp. 204-207. 
linear variety defined by $L(u)=0$ in the vector-space of dimension $r+1$ over $k$.

If $k^{*}$ is the multiplicative group of all non-zero elements in $k$, we shall denote by the letter $\chi$ any character of $k^{*}$; as $k^{*}$ is cyclic of order $q-1$, such a character is fully determined if one assigns its value at a generating element $w$ of $k^{*}$ (a "primitive root"), and this value may be any $(q-1)$ th root of unity. Selecting such an element $w$ once for all, we shall denote by $\chi_{\alpha}$ the character of $k^{*}$ determined by $\chi_{\alpha}(w)=e^{2 \pi i \alpha}$, where $\alpha$ is a rational number satisfying $(q-1) \alpha \equiv 0$ $(\bmod 1)$. We also put $\chi_{\alpha}(0)=0$ for $\alpha \neq \equiv(\bmod 1)$ and $\chi_{\alpha}(0)=1$ for $\alpha \equiv 0(\bmod 1)$. Then we have

$$
N_{i}(u)=\sum_{\alpha} \chi_{\alpha}(u) \quad\left(d_{i} \alpha \equiv 0(\bmod 1), 0 \leqq \alpha<1\right) .
$$

In fact, for $u=0$, both sides have the value 1 ; for $u \neq 0$, the righthand side can be written as $\sum_{\nu=0}^{d_{i}-1} \zeta^{\nu}$, with $\zeta=\chi_{1 / d_{i}}(u)$; and $\zeta$ is then a $d_{i}$ th root of unity, equal to 1 if and only if $u$ is a $d_{i}$ th power in $k^{*}$.

Using this in (2), we get:

$$
\begin{aligned}
& N=\sum_{u, \alpha} \chi_{\alpha_{0}}\left(u_{0}\right) \cdots \chi_{\alpha_{r}}\left(u_{r}\right) \\
& \quad\left(L(u)=0 ; d_{i} \alpha_{i} \equiv 0(\bmod 1), 0 \leqq \alpha_{i}<1\right) .
\end{aligned}
$$

As there are $q^{r}$ points in $L(u)=0$, the terms in the above sum which correspond to $\alpha_{0}=\cdots=\alpha_{r}=0$, being all equal to 1 , give a sum $q^{r}$. We now show that those terms for which some, but not all, of the $\alpha_{i}$ are 0 , give a sum 0 . In fact, consider e.g. those for which $\alpha_{0}, \cdots, \alpha_{s-1}$ have given values, other than 0 , and $\alpha_{s}=\cdots=\alpha_{r}=0$, with $s \leqq r$; as there are $q^{r-s}$ points $(u)$ in the variety $L(u)=0$ for which $u_{0}, \cdots$, $u_{s-1}$ have arbitrarily assigned values, the sum of those terms is

$$
q^{r-s} \prod_{i=0}^{s-1}\left(\sum_{u_{i}} \chi_{\alpha_{i}}\left(u_{i}\right)\right)
$$

and this is 0 since each factor is 0 . This gives

$$
\begin{gathered}
N=q^{r}+\sum_{u, \alpha} \chi_{\alpha_{0}}\left(u_{0}\right) \cdots \chi_{\alpha_{r}}\left(u_{r}\right) \\
\left(L(u)=0 ; d_{i} \alpha_{i} \equiv 0(\bmod 1), 0<\alpha_{i}<1\right) .
\end{gathered}
$$

In this, we replace the $u_{i}$, respectively, by $u_{i} / a_{i}$, and get

$$
\begin{aligned}
N=q^{r}+\sum_{\alpha} \chi_{\alpha_{0}}\left(a_{0}^{-1}\right) & \cdots \chi_{\alpha_{r}}\left(a_{r}^{-1}\right) \cdot S(\alpha) \\
& \left(d_{i} \alpha_{i} \equiv 0(\bmod 1), 0<\alpha_{i}<1\right),
\end{aligned}
$$

if we put, for any values of $\alpha_{i}$ satisfying $(q-1) \alpha_{i} \equiv 0(\bmod 1), \alpha_{i} \neq 0$ 
$(\bmod 1):$

$$
S(\alpha)=S\left(\alpha_{0}, \cdots, \alpha_{r}\right)=\sum_{\Sigma u_{i}=0} \chi_{\alpha_{0}}\left(u_{0}\right) \cdots \chi_{\alpha_{r}}\left(u_{r}\right)
$$

As to the latter sum, the terms for which $u_{0}=0$ are 0 , and we may exclude them; we may then put $u_{i}=u_{0} v_{i}(1 \leqq i \leqq r)$; the terms, in our sum, corresponding to given values of the $v_{i}$ (satisfying $1+\sum_{i=1}^{r} v_{i}=0$ ) give

$$
\chi_{\alpha_{1}}\left(v_{1}\right) \cdots \chi_{\alpha_{r}}\left(v_{r}\right) \sum_{u_{0} \neq 0} \chi_{\beta}\left(u_{0}\right)
$$

with $\beta=\sum_{i=0}^{r} \alpha_{i}$, and this last sum is $q-1$ for $\beta \equiv 0(\bmod 1)$, and 0 otherwise, so that in the latter case $S(\alpha)$ is 0 .

Let us therefore define, for any set of $\alpha_{i}$ satisfying the conditions

$$
(q-1) \alpha_{i} \equiv 0, \quad \alpha_{i} \neq \equiv 0, \quad \sum_{i=0}^{r} \alpha_{i} \equiv 0(\bmod 1),
$$

a number $j(\alpha)$ by the relation

$$
\begin{aligned}
j(\alpha) & =\sum_{1+v_{1}+\cdots+v_{r}=0} \chi_{\alpha_{1}}\left(v_{1}\right) \cdots \chi_{\alpha_{r}}\left(v_{r}\right) \\
& =\frac{1}{q-1} \sum_{u_{0}+\cdots+u_{r}=0} \chi_{\alpha_{0}}\left(u_{0}\right) \cdots \chi_{\alpha_{r}}\left(u_{r}\right) .
\end{aligned}
$$

In terms of the $j(\alpha)$, the number $N$ of solutions of $\sum_{i=0}^{r} a_{i} x_{i}^{n}=0$ is now seen to be given by

$$
\begin{aligned}
N=q^{r}+(q-1) & \sum_{\alpha} \chi_{\alpha_{0}}\left(a_{0}^{-1}\right) \cdots \chi_{\alpha_{r}}\left(a_{r}^{-1}\right) \cdot j(\alpha) \\
& \left(d_{i} \alpha_{i} \equiv 0 ; \sum \alpha_{i} \equiv 0(\bmod 1) ; 0<\alpha_{i}<1\right) .
\end{aligned}
$$

The $j(\alpha)$ may be called the Jacobi sums for the field $k$; they were first introduced and studied, for the case of a prime field, by Jacobi [2a, b], later by Stickelberger [7], and more recently by Davenport and Hasse [5]. They are closely related to the Gaussian sums for $k$ :

$$
g(\chi)=\sum_{x \in k} \chi(x) \psi(x)
$$

where $\psi$ is a character of the additive group of $k$, chosen once for all, and not everywhere equal to 1 , and where $\chi$ is any one of the above defined multiplicative characters, other than $\chi_{0}$. For the convenience of the reader, we shall briefly recall some of the known properties of these sums. In the first place, in the sum which defines $g(\chi)$, we may, as $\chi$ is not $\chi_{0}$, restrict $x$ to be $\neq 0$. Then we get 


$$
g(\chi) \bar{g}(\chi)=\sum_{y \neq 0} \sum_{x \neq 0} \chi\left(x y^{-1}\right) \psi(x-y),
$$

where we may substitute $x y$ for $x$ in the sum for $x$, and then interchange the order of summations:

$$
g(\chi) \bar{g}(\chi)=\sum_{x \neq 0} \chi(x) \sum_{y \neq 0} \psi[(x-1) y]
$$

As the sum of all values of $\psi$ on $k$ is 0 , the second sum has the value $q-1$ for $x=1$, and -1 for $x \neq 1$; as the sum of all values of $\chi$ on $k^{*}$ is 0 , this gives

$$
g(\chi) \bar{g}(\chi)=q
$$

Now, in the definition of $g(\chi)$, write $t x$ for $x$ with any $t \neq 0$ in $k$; this gives

$$
g(\chi)=\chi(t) \sum_{x} \chi(x) \psi(t x)
$$

hence, using (4), and interchanging $x$ and $t$ :

$$
\chi(x)=\frac{g(\chi)}{q} \sum_{t} \bar{\chi}(t) \bar{\psi}(t x),
$$

which is also true for $x=0$; this is the Fourier expansion of $\chi(x)$ on $k$ according to the additive characters of $k$. Using this in the definition of $j(\alpha)$, we get

$$
\begin{array}{r}
(q-1) j(\alpha)=q^{-r-1} \cdot g\left(\chi_{\alpha_{0}}\right) \cdots g\left(\chi_{\alpha_{r}}\right) \\
\sum_{t} \bar{\chi}_{\alpha_{0}}\left(t_{0}\right) \cdots \bar{\chi}_{\alpha_{r}}\left(t_{r}\right) \\
\sum_{\Sigma u_{i}=0} \Psi\left(\sum_{i} t_{i} u_{i}\right) .
\end{array}
$$

But, in the additive group of all vectors $(u)=\left(u_{0}, \cdots, u_{r}\right)$, the vectors satisfying $\sum u_{i}=0$ form a subgroup of $q^{r}$ elements, on which $\Psi\left(\sum t_{i} u_{i}\right)$ is a character; the sum of the values of this character on the subgroup must therefore be either $q^{r}$, if the character has the constant value 1 , or 0 otherwise. The former case occurs if and only if all the $t_{i}$ are equal, since otherwise we can solve the equations $\sum u_{i}=0, \sum t_{i} u_{i}=z$, where $z$ is any element of $k$, e.g. one such that $\psi(z) \neq 1$. As we have $\sum \alpha_{i} \equiv 0(\bmod 1)$ by the definition of $j(\alpha)$, this gives

$$
\mathcal{L}^{(\alpha)}=\frac{1}{q} g\left(\chi_{\alpha_{0}}\right) \cdots g\left(\chi_{\alpha_{r}}\right) .
$$


As a consequence, we have

$$
j(\alpha) j(\alpha)=q^{r-1}
$$

and therefore

$$
\left|N-q^{r}\right| \leqq M(q-1) q^{(r-1) / 2},
$$

where $M$ is the number of systems of rational numbers $\alpha_{i}$ satisfying

$$
n_{i} \alpha_{i} \equiv 0, \sum \alpha_{i} \equiv 0(\bmod 1), 0<\alpha_{i}<1,
$$

and is therefore an integer depending only upon the $n_{i}$.

From the above results, we can easily derive the number $N_{1}$ of solutions of the equation $\sum_{i=0}^{r} a_{i} x_{i}^{n_{i}}+1=0$. In fact, let $N$, as before, be the number of solutions of $\sum_{i=0}^{r} a_{i} x_{i}^{n_{i}}=0$, and let $N^{\prime}$ be the number of solutions of $\sum_{i=0}^{r} a_{i} x_{i}^{n_{i}}+x_{r+}^{q-1}=0$. The previous results apply to the latter equation, with $d_{r+1}=n_{r+1}=q-1$. But, since $x_{r+1}^{q-1}$ has the value 1 , except for $x_{r+1}=0$, we have

$$
N^{\prime}=(q-1) N_{1}+N
$$

This gives at once an expression for $N_{1}$; in order to write it more conveniently, we shall define the symbol $j(\alpha)$ even in the case when some, but not all, of the $\alpha_{i}$ are 0 . Let the $\beta_{j}$ be numbers, satisfying $(q-1) \beta_{j}$ $\equiv 0, \sum_{j} \beta_{j} \equiv 0(\bmod 1)$, and not all $\equiv 0(\bmod 1)$; assume that $s$ of them are $\equiv 0(\bmod 1)$, and let $\alpha_{0}, \cdots, \alpha_{r}$ be the others, in any order; then we put $j(\beta)=(-1)^{s} j(\alpha)$. This being so, the formula for $N_{1}$ can be written as

$$
\begin{array}{r}
N_{1}=q^{r}+\sum_{\alpha} \chi_{\alpha_{0}}\left(a_{0}^{-1}\right) \cdots \chi_{\alpha_{r}}\left(a_{r}^{-1}\right) j\left(\alpha_{0}, \cdots, \alpha_{r},-\sum_{i=0}^{r} \alpha_{i}\right) \\
\left(d_{i} \alpha_{i} \equiv 0(\bmod 1), 0<\alpha_{i}<1\right),
\end{array}
$$

and we get, as before:

$$
\left|N_{1}-q^{r}\right| \leqq M_{1} q^{r / 2}
$$

where $M_{1}$ is now given by

$$
M_{1}=\left(d_{0}-1\right) \cdots\left(d_{r}-1\right)<n_{0} n_{1} \cdots n_{r} .
$$

It is a matter of considerable interest to be able to compare the number of solutions of an equation (or, more generally, the number of rational points on an algebraic variety) in a given finite field and in all the extensions of finite degree of that field. This can easily be done, for the type of equations under consideration in this note, if we use a relation, due to Davenport and Hasse [5], between Gaussian 
sums in a finite field and in its extensions. We shall first give a brief account, in elementary language, of the proof of Davenport and Hasse for this relation.

Let $k^{\prime}$ be an extension of $k$, of degree $\nu$; for $y$ in $k^{\prime}$, let $N(y)$ and $T(y)$ denote the norm and the trace of $y$, respectively, over $k$. If $w$ denotes, as before, a generator of the multiplicative group $k^{*}$, there is a generator $z$ of $k^{* *}$, such that $N(z)=w$; then, if we denote, as before, by $\chi_{\alpha}^{\prime}(y)$ the multiplicative character on $k^{\prime}$ determined by $\chi_{\alpha}^{\prime}(z)=e^{2 \pi i \alpha}$, we have, for $(q-1) \alpha \equiv 0(\bmod 1), \chi_{\alpha}^{\prime}(y)=\chi_{\alpha}[N(y)]$. We also put $\psi^{\prime}(y)=\psi[T(y)]$; this is an additive character of $k^{\prime}$, not everywhere equal to 1 since it is known that $T(y)$ maps $k^{\prime}$ on $k$. Let now $g^{\prime}\left(\chi_{\alpha}^{\prime}\right)$ be the Gaussian sum in $k^{\prime}$ :

$$
g^{\prime}\left(\chi_{\alpha}^{\prime}\right)=\sum_{y \in k^{\prime}} \chi_{\alpha}^{\prime}(y) \psi^{\prime}(y) .
$$

The theorem of Davenport and Hasse is as follows:

$$
-g^{\prime}\left(\chi_{\alpha}^{\prime}\right)=\left[-g\left(\chi_{\alpha}\right)\right]^{\nu} .
$$

In order to prove this, consider the polynomials with coefficients in $k$, and highest coefficient 1 ; to every such polynomial

$$
F(X)=X^{n}+c_{1} X^{n-1}+\cdots+c_{n},
$$

of degree $n \geqq 1$, we attach the number

$$
\lambda(F)=\chi_{\alpha}\left(c_{n}\right) \psi\left(c_{1}\right) .
$$

For two such polynomials $F_{1}, F_{2}$, we have $\lambda\left(F_{1} F_{2}\right)=\lambda\left(F_{1}\right) \lambda\left(F_{2}\right)$. If we also denote by $n(F)$ the degree of such a polynomial $F$, and by $U$ an indeterminate, this gives the formal identity

$$
1+\sum_{F} \lambda(F) \cdot U^{n(F)}=\prod_{P}\left[1-\lambda(P) \cdot U^{n(P)}\right]^{-1},
$$

where the sum in the left-hand side is taken over all polynomials $F$ over $k$, of degree $\geqq 1$, with highest coefficient 1 , and the product in the right-hand side is taken over all irreducible polynomials $P$ over $k$, with highest coefficient 1 . As usual, this follows at once from the fact that every $F$ can be expressed in a unique manner as product of powers of irreducible polynomials.

In the sum in the left-hand side, consider first the terms which correspond to polynomials $F(X)=X+c$ of degree 1 ; the sum of these terms is equal to $g\left(\chi_{\alpha}\right) U$. As to the sum of the terms corresponding to any given degree $n>1$, it is 0 , since, with the above notations, it is equal to 


$$
q^{n-2} \sum_{c_{n}} \chi_{\alpha}\left(c_{n}\right) \sum_{c_{1}} \psi\left(c_{1}\right) \cdot U^{n}
$$

where both sums are taken over $k$ and are therefore 0 . This gives

$$
1+g\left(\chi_{\alpha}\right) U=\prod_{P}\left[1-\lambda(P) \cdot U^{n(P)}\right]^{-1} .
$$

Similarly, if $F^{\prime}(X)=X^{n}+d_{1} X^{n-1}+\cdots+d_{n}$ is a polynomial over $k^{\prime}$, we write

$$
\lambda^{\prime}\left(F^{\prime}\right)=\chi_{\alpha}^{\prime}\left(d_{n}\right) \psi^{\prime}\left(d_{1}\right),
$$

and, taking another indeterminate $U^{\prime}$, get the formal identity

$$
1+g^{\prime}\left(\chi_{\alpha}^{\prime}\right) U^{\prime}=\prod_{P^{\prime}}\left[1-\lambda^{\prime}\left(P^{\prime}\right) \cdot U^{\prime n\left(P^{\prime}\right)}\right]^{-1}
$$

where the product is taken over all irreducible polynomials $P^{\prime}$ over $k^{\prime}$, with highest coefficient 1.

Now let $P$ be as above; let $P^{\prime}$ be one of the irreducible factors of $P$ over $k^{\prime}$; let $-\xi$ be one of the roots of $P^{\prime}$. Then $\xi$ generates over $k$ an extension $k(\xi)$ of degree $n=n(P)$, and over $k^{\prime}$ an extension $k^{\prime}(\xi)$ of degree $n^{\prime}=n\left(P^{\prime}\right)$; as $k^{\prime}(\xi)$ is the composite of $k(\xi)$ and $k^{\prime}$, its degree over $k$ must be the 1.c.m. of the degree $n$ of $k(\xi)$ over $k$, and of the degree $\nu$ of $k^{\prime}$ over $k$, i.e. equal to $n \nu / d$ if we write $d=(n, \nu)$. This gives $n^{\prime}=n / d$; hence $P$ has over $k^{\prime}$ exactly $d$ irreducible factors, all of degree $n / d$. Moreover, if $a$ and $b$ are respectively the norm and the trace of $\xi$, taken in $k(\xi)$ relatively to $k$, we have

$$
P(X)=X^{n}+b X^{n-1}+\cdots+a,
$$

hence

$$
\lambda(P)=\chi_{\alpha}(a) \psi(b) .
$$

Similarly, if $a^{\prime}$ and $b^{\prime}$ are the norm and the trace of $\xi$, taken in $k^{\prime}(\xi)$ relatively to $k^{\prime}$, we have

$$
\lambda^{\prime}\left(P^{\prime}\right)=\chi_{\alpha}^{\prime}\left(a^{\prime}\right) \psi^{\prime}\left(b^{\prime}\right)=\chi_{\alpha}\left(N a^{\prime}\right) \psi\left(T b^{\prime}\right),
$$

where $N a^{\prime}$ and $T b^{\prime}$ are the norm of $a^{\prime}$ and the trace of $b^{\prime}$, taken in $k^{\prime}$ relatively to $k$; hence $N a^{\prime}$ and $T b^{\prime}$ are respectively equal to the norm and to the trace of $\xi$, taken in $k^{\prime}(\xi)$ relatively to $k$. We can therefore also obtain $N a^{\prime}$ by taking the norm of $\xi$ in $k^{\prime}(\xi)$ relatively to $k(\xi)$, this being equal to $\xi^{\nu / d}$, and then the norm of this in $k(\xi)$ relatively to $k$, which is $a^{\nu / d}$. Hence we have $N a^{\prime}=a^{\nu / d}$, and similarly $T b^{\prime}=(\nu / d) b$, and therefore

$$
\lambda^{\prime}\left(P^{\prime}\right)=\lambda(P)^{\nu / d}
$$


Now, in the right-hand side of $\left(6^{\prime}\right)$, we can put together the $d$ factors corresponding to all the irreducible factors of $P$ over $k^{\prime}$; if, moreover, we replace $U^{\prime}$ by $U^{p}$, we get

$$
\left[1-\lambda(P)^{\nu / d} U^{v n / d}\right]^{-d},
$$

which can also be written as

$$
\prod_{\rho=0}^{\nu-1}\left[1-\lambda(P) \cdot\left(\zeta^{\rho} U\right)^{n}\right]^{-1}
$$

where $\zeta$ is any primitive $\nu$ th root of unity. This gives

$$
\begin{aligned}
1+g^{\prime}\left(\chi_{\alpha}^{\prime}\right) U^{\nu} & =\prod_{\rho=0}^{\nu-1} \prod_{P}\left[1-\lambda(P) \cdot\left(\zeta^{\rho} U\right)^{n(P)}\right]^{-1} \\
& =\prod_{\rho=0}^{\nu-1}\left(1+g\left(\chi_{\alpha}\right) \zeta^{\rho} U\right) \\
& =1+(-1)^{\nu+1} g\left(\chi_{\alpha}\right)^{\nu} U^{\nu},
\end{aligned}
$$

which proves (5).

Now, $N_{\nu}$ being the number of solutions of an equation of type (1), with or without constant term, over the extension of degree $\nu$ of the ground-field $k$, it is easy, using the above results, to give a simple expression for the "generating power-series" for $N_{\nu}$, i.e. for the formal power-series $\sum_{1}^{\infty} N_{\nu} U^{\nu}$; this turns out to be the expansion of a certain rational function in $U$. We shall, however, illustrate this idea by considering the case of the homogeneous equation

$$
a_{0} x_{0}^{n}+\cdots+a_{r} x_{r}^{n}=0,
$$

considered as the equation of a variety (without singular points) in the projective space $P^{r}$ of dimension $r$ over $k$. The number $\bar{N}$ of rational points over $k$, on that variety, is related to the number $N$ of solutions of the same equation in affine space by $N=1+(q-1) \bar{N}$, so that, putting $d=(n, q-1)$, we get, from our earlier results:

$$
\begin{aligned}
\bar{N}=1+q+\cdots+q^{r-1} & +\sum_{\alpha} \bar{\chi}_{\alpha_{0}}\left(a_{0}\right) \cdots \bar{\chi}_{\alpha_{r}}\left(a_{r}\right) \cdot j(\alpha) \\
\left(d \alpha_{i}\right. & \left.\equiv 0, \sum \alpha_{i} \equiv 0(\bmod 1) ; 0<\alpha_{i}<1\right) .
\end{aligned}
$$

Now call $\bar{N}_{\nu}$ the number of rational points, on the variety defined by (7), over the extension $k_{\nu}$ of $k$ of degree $\nu$; we shall calculate the series $\sum_{1}^{\infty} \bar{N}_{\nu} U^{\nu-1}$.

In order to do this, consider any set of rational numbers $\alpha_{0}, \cdots, \alpha_{r}$ satisfying $n \alpha_{i} \equiv 0, \sum \alpha_{i} \equiv 0(\bmod 1), 0<\alpha_{i}<1$. For this set, let 
$\mu=\mu(\alpha)$ be the smallest integer such that $\left(q^{\mu}-1\right) \alpha_{i} \equiv 0(\bmod 1)$ for $0 \leqq i \leqq r$; then the extensions $k_{\nu}$ of $k$ such that $\left(q^{\nu}-1\right) \alpha_{i} \equiv 0(\bmod 1)$ are those for which $\nu$ is a multiple of $\mu$, and those only. Choosing a primitive root in $k_{\mu}$, we can now, as before, define in $k_{\mu}$ the characters $\chi_{\alpha_{i}}$, the Gaussian sums $g\left(\chi_{\alpha_{i}}\right)$, and the Jacobi sum

$$
j(\alpha)=\frac{1}{q} g\left(\chi_{\alpha_{0}}\right) \cdots g\left(\chi_{\alpha_{r}}\right) .
$$

Furthermore, if we denote by $\chi_{\alpha_{i}}^{\prime}, g^{\prime}\left(\chi_{\alpha_{i}}^{\prime}\right)$ and $j^{\prime}(\alpha)$ the corresponding characters and sums for the extension $k^{\prime}=k_{\lambda \mu}$ of $k$ of degree $\lambda \mu$, where $\lambda$ is any integer, we get from our earlier results:

$\chi_{\alpha_{i}}^{\prime}\left(a_{i}\right)=\chi_{\alpha_{i}}\left(a_{i}\right)^{\lambda}, g^{\prime}\left(\chi_{\alpha_{i}}^{\prime}\right)=(-1)^{\lambda-1} g\left(\chi_{\alpha_{i}}\right)^{\lambda}, j^{\prime}(\alpha)=(-1)^{(\lambda-1)(r-1)} j(\alpha)^{\lambda}$.

Then we get:

$$
\begin{aligned}
\sum_{1}^{\infty} \bar{N}_{\nu} U^{\nu-1}= & -\sum_{h=0}^{r-1} \frac{d}{d U} \log \left(1-q^{h} U\right) \\
& +(-1)^{r} \sum_{\alpha} \frac{1}{\mu(\alpha)} \frac{d}{d U} \log \left[1-C(\alpha) \cdot U^{\mu(\alpha)}\right] \\
& \left(n \alpha_{i} \equiv 0, \sum \alpha_{i} \equiv 0(\bmod 1) ; 0<\alpha_{i}<1\right)
\end{aligned}
$$

where we have put

$$
C(\alpha)=(-1)^{r-1} \bar{\chi}_{\alpha_{0}}\left(a_{0}\right) \cdots \bar{\chi}_{\alpha_{r}}\left(a_{r}\right) \cdot j(\alpha) .
$$

Furthermore, it is easily seen that $C(q \alpha)=C(\alpha)$, since $x \rightarrow x^{q}$ is an automorphism of $k_{\mu}$ which leaves the $a_{i}$ invariant. Therefore, in the last sum in (8), the $\mu(\alpha)$ terms corresponding to the set $(\alpha)=\left(\alpha_{0}, \cdots, \alpha_{r}\right)$ and to the sets $\left(q^{\rho} \alpha\right)$ for $1 \leqq \rho \leqq \mu-1$ are all equal, so that, putting them together, we can make the denominator $\mu(\alpha)$ disappear.

Let $A$ be the number of solutions, in rational numbers $\alpha_{i}$, of the system $n \alpha_{i} \equiv 0, \sum \alpha_{i} \equiv 0(\bmod 1), 0<\alpha_{i}<1$. Then one finds ${ }^{4}$ that the Poincare polynomial (in the sense of combinatorial topology) of the variety defined, in the projective space $P^{r}$ over complex numbers, by an equation of the form

$$
c_{0} x_{0}^{n}+\cdots+c_{r} x_{r}^{n}=0
$$

is equal to

${ }^{4}$ As obligingly communicated to me by P. Dolbeault in Paris. 


$$
\sum_{h=0}^{r-1} X^{2 h}+A \cdot X^{r-1}
$$

This, and other examples which we cannot discuss here, seem to lend some support to the following conjectural statements, which are known to be true for curves, but which I have not so far been able to prove for varieties of higher dimension.

Let $V$ be a variety without singular points, of dimension $n$, defined over a finite field $k$ with $q$ elements. Let $N_{\nu}$ be the number of rational points on $V$ over the extension $k_{\nu}$ of $k$ of degree $\nu$. Then we have

$$
\sum_{1}^{\infty} N_{\nu} U^{\nu-1}=\frac{d}{d U} \log Z(U)
$$

where $Z(U)$ is a rational function in $U$, satisfying a functional equation

$$
Z\left(\frac{1}{q^{n} U}\right)= \pm q^{n x / 2} U^{x} Z(U),
$$

with $\chi$ equal to the Euler-Poincaré characteristic of $V$ (intersectionnumber of the diagonal with itself on the product $V \times V$ ).

Furthermore, we have:

$$
Z(U)=\frac{P_{1}(U) P_{3}(U) \cdots P_{2 n-1}(U)}{P_{0}(U) P_{2}(U) \cdots P_{2 n}(U)},
$$

with $P_{0}(U)=1-U, P_{2 n}(U)=1-q^{n} U$, and, for $1 \leqq h \leqq 2 n-1$ :

$$
P_{h}(U)=\prod_{i=1}^{B_{h}}\left(1-\alpha_{h i} U\right)
$$

where the $\alpha_{h i}$ are algebraic integers of absolute value $q^{h / 2}$.

Finally, let us call the degrees $B_{h}$ of the polynomials $P_{h}(U)$ the Betti numbers of the variety $V$; the Euler-Poincaré characteristic $\chi$ is then expressed by the usual formula $\chi=\sum_{h}(-1)^{h} B_{h}$. The evidence at hand seems to suggest that, if $\bar{V}$ is a variety without singular points, defined over a field $K$ of algebraic numbers, the Betti numbers of the varieties $V_{\mathfrak{p}}$, derived from $\bar{V}$ by reduction modulo a prime ideal $\mathfrak{p}$ in $K$, are equal to the Betti numbers of $\bar{V}$ (considered as a variety over complex numbers) in the sense of combinatorial topology, for all except at most a finite number of prime ideals $\mathfrak{p}$. For instance, consider the Grassmann variety $G_{m, r}$, the points of which are the $r$-dimensional linear varieties in a projective $m$-dimensional space, over 
a field with $q$ elements. The number of rational points on the variety is easily seen to be $F(q)$, where $F$ is the polynomial defined by

$$
F(X)=\frac{\left(X^{m+1}-1\right)\left(X^{m+1}-X\right) \cdots\left(X^{m+1}-X^{r}\right)}{\left(X^{r+1}-1\right)\left(X^{r+1}-X\right) \cdots\left(X^{r+1}-X^{r}\right)} .
$$

Then, if the above conjectures are true, the Poincaré polynomial of the Grassmann variety $G_{m, r}$ over complex numbers must be $F\left(X^{2}\right)$. This is indeed so, as can easily be verified from the well-known results of Ehresmann [8]. 5

\section{BIBLIOGRAPHY}

1. C. F. Gauss, Werke: (a) vol. I, pp. 445-449; (b) vol. II, pp. 67-92; (c) vol. $\mathrm{X}_{1}$, p. 571 .

2. C. G. Jacobi, Gesammelte Werke: (a) vol. VII, pp. 393-400; (b) vol. VI, pp. 254-274.

3. V. A. Lebesgue: (a) J. Math. Pures Appl. vol. 2 (1837) pp. 253-292; (b) J. Math. Pures Appl. vol. 3 (1838) pp. 113-144.

4. G. H. Hardy and J. E. Littlewood, Math. Zeit. vol. 12 (1922) pp. 161-188.

5. H. Davenport and H. Hasse, J. Reine Angew. Math. vol. 172 (1935) pp. 151182.

6. L. K. Hua and H. S. Vandiver, Proc. Nat. Acad. Sci. U.S.A. vol. 34 (1948) pp. 258-263.

7. L. Stickelberger, Math. Ann. vol. 37 (1890) pp. 321-367.

8. Ch. Ehresmann, Ann. of Math. vol. 35 (1934) pp. 396-443.

The University of Chiçago

5 Added in proof. Results, substantially identical to our formula (3), have just been published by L. K. Hua and H. S. Vandiver, Proc. Nat. Acad. Sci. U.S.A. vol. 35 (1949) pp. 94-99. 